\title{
P/Halley: The Disconnection Event of 1986 April 11
}

\author{
P.M. Bergé ${ }^{1}$, G. Mahoux ${ }^{2}$ and A.C. Levasseur-Regourd ${ }^{3}$ \\ ${ }^{1}$ Residence "La Dauniere" Bat. D, F-91940 Les Ulis, France \\ 212 rue du Dr. Kurzenne, F-78350 Jouy-en-Josas, France \\ ${ }^{3}$ Université Paris VI, Service d'Aeronomie, F-91370 Verrières-le-Buisson, France
}

This work was carried out by an expedition organized by the S.A.F. in 1986 April to La Réunion. The aim was to observe and photograph P/Halley, within the framework of IHW, as part of the Island Network in the southern hemisphere. To be more precise, our work consisted of studying large-scale phenomena: the structure, dynamics and possible disconnection events in the plasma tail. We were lucky enough to observe one of the latter on the night of April 11/12, and describe it here.

For the Island Network, IHW had a number of Schmidt telescopes (Celestron 8). One was lent to the S.A.F. and this is what we used. This telescope has a focal ratio of 1.5 , with a $200-\mathrm{mm}$ (8-inch) objective and $300-\mathrm{mm}$ focal length. We used only Kodak TP 2415 film, hypersensitized in forming gas $\left(24 \mathrm{~h}\right.$ at $\left.60^{\circ} \mathrm{C}\right)$.

We obtained three photographs of the discontinuity, only the last two being reproduced here (as negatives). The guiding was on the comet's central condensation. In addition, 3 other photographs were available, taken by Gerhart Klaus in the Namib Desert, using a Schmidt similar to ours (but outside the IHW framework).

\begin{tabular}{lcccc}
\hline Photo. No. Site & Date (UT) & Exposure & Filter \\
\hline 1 & La Réunion & Apr.11.868 & $9 \mathrm{~m}$ & none \\
2 & \# & Apr.11.881 & $20 \mathrm{~m}$ & $"$ \\
3 & $"$ & Apr.11.928 & $60 \mathrm{~m}$ & W47B \\
4 & Namibia & Apr.11.889 & $10 \mathrm{~m}$ & none \\
5 & $"$ & Apr.11.920 & $10 \mathrm{~m}$ & $"$ \\
6 & $"$ & Apr.12.090 & $10 \mathrm{~m}$ & $"$ \\
\hline
\end{tabular}

On photo 2, the coma, the rather wide dust tail, and straighter plasma tail, which shows a very distinct disconnection near the coma, can all be seen. On photo 3 , the star trails are much longer, the exposure being 60 minutes. The blue W47B filter has practically extinguished the yellow-coloured dust tail, but retained the bluish plasma tail. In the old tail, emitted before the disconnection, parallel structures can be seen, which suggest the existence of turbulence in this part of the plasma tail. The last photograph taken by G. Klaus about 4 hours after out third photograph (and not reproduced here), shows considerable evolution in the plasma tail, near the disconnection. 
Reduction of the photographs required the calculation for each of them of the equatorial coordinates of the comet, the $\Delta$ and $\mathrm{R}$ distances from the Earth and the Sun, the antisolar direction, and the phase angle (the Earth-comet-Sun angle, necessary for the measurement of real distances along the plasma tail). We used the photographic atlas by Papadopoulos and Scovil to determine the position of the cometary nucleus on each plate, as well as the direction of North and the antisolar direction, which allowed us to measure the tail's directions before and after the disconnection. We measured the speed of recession of two specific points on the plasma tail: the one where the old tail became disconnected, and the one where the new tail reformed. We found $50 \pm 5$ and $80 \pm 20 \mathrm{~km} / \mathrm{s}$ respectively, which agree with the values usually found for the recession velocities of cometary tails. Despite the error in the measurements, caused by the difficulty in identifying exactly the same points on the differnet plates, the velocity of the new tail seems significantly greater than that of the older one.

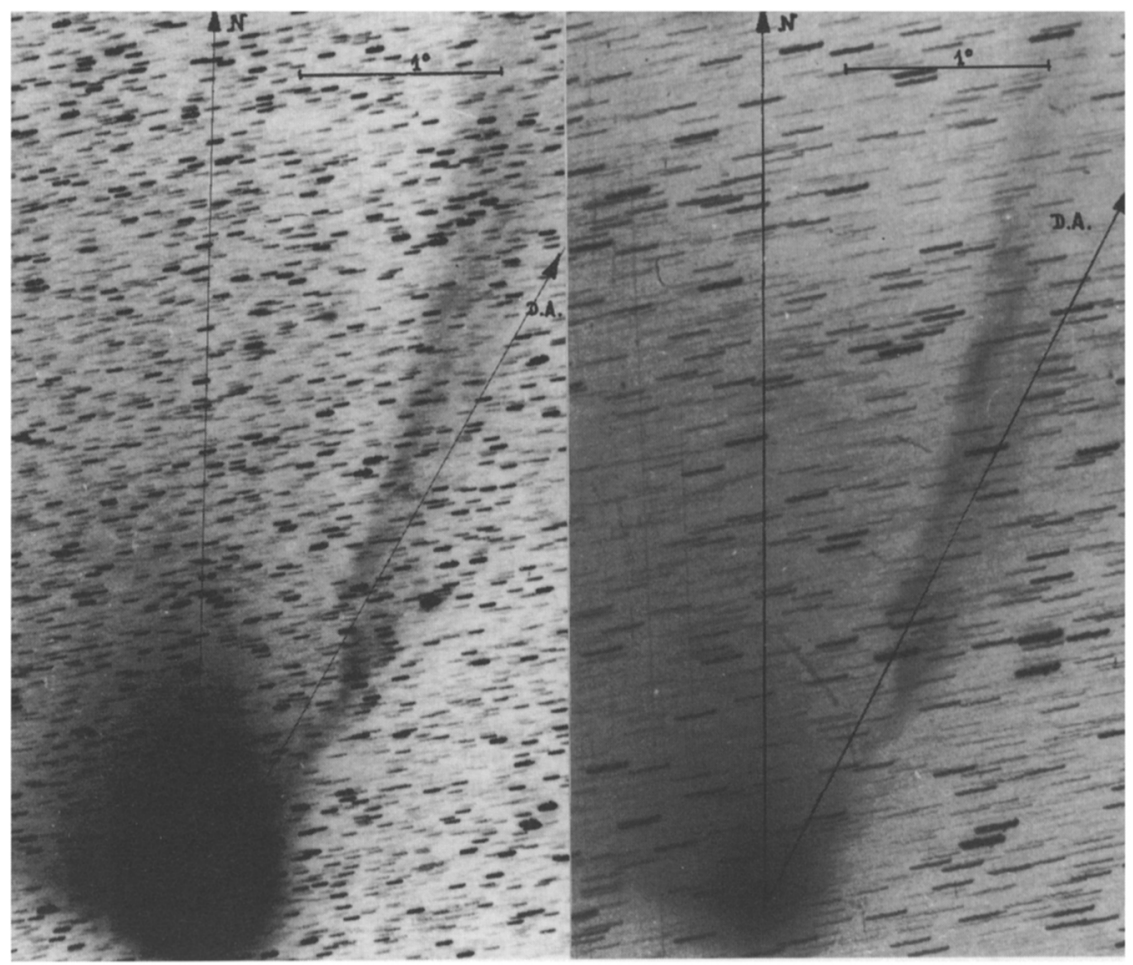

Photos 2 (left) and 3 (right)

The interpretation currently given for disconnection events, proposed by Malcolm B. Niedner and John C. Brandt (1), calls for the comet to pass through a region where the magnetic field carried by the solar wind changes sense. It is known that the ions of cometary origin that form the plasma tail are channeled along the lines of magnetic force. In a region where the field rapidly changes its sense, the plasma 
tail is dislocated, and a new tail forms slightly later, when the new magnetic field has stabilized. However, as far as the event that we are considering here is concerned, Niedner himself has cast grave doubt on this explanation (2). On April 11, the comet would not have crossed a boundary between magnetic-field sectors of opposite polarity. However, the interplanetary probes IMP-8, Pioneer Venus Orbiter and ICE have revealed the existence of small-scale reversals of polarity outside the neutral sheet, where such reversals are normally expected. This could be the cause of the April 11 event, which would therefore be a sign of local turbulence in the solar wind and its magnetic field.

\section{References}

1. M.B. Niedner, J.C. Brandt: Astrophys. J., 223, 655 (1978)

2. M.B. Niedner: COSPAR Meeting, Toulouse, France, 1986 July 